\title{
Asymmetric Polymerization of Isocyanates with Optically Active Anionic Initiators
}

\author{
Yoshio OKamoto, Masashi Matsuda, Tamaki NaKano, \\ and Eiji YASHIMA \\ Department of Applied Chemistry, Faculty of Engineering, \\ Nagoya University, Furo-cho, Chikusa-ku, \\ Nagoya 464-01, Japan
}

(Received September 22, 1992)

\begin{abstract}
Anionic polymerization of 3-phenylpropyl, 4-phenylbutyl, and butyl isocyanates was carried out with chiral anionic initiators such as tert-butyllithium- $(-)$-sparteine complex $(t$-BuLi- $(-)-\mathrm{Sp})$, lithium (-)-menthoxide, and (+)-1-(2-pyrrolidinylmethyl)pyrrolidine lithium amide. The $t$-BuLi- $(-)$-Sp did not afford optically active polymers. However, the alkoxide and amide gave optically active polymers for 4-phenylbutyl and butyl isocyanates. The optical activities are probably based on the prevailing helicity of the polymer chains induced by chiral initiator residues attaching to an $\alpha$-end group of the polymer chain. The optical rotation of the polymers depended greatly on molecular weight and temperature.
\end{abstract}

KEY WORDS Polyisocyanate / Anionic Polymerization / Helix / Optically Active Polymer / $\omega$-Phenylalkyl Isocyanate /

Polyisocyanates have a rigid rodlike main chain based on the structure of 1-nylon and have extensively been studied from the viewpoints of synthesis, structure, and character. ${ }^{1,2}$ Several polyisocyanates with optically active side groups have been prepared mainly because the polyisocyanates show interesting chiroptical properties due to helical conformation. $^{3-8}$ Most polyisocyanates so far investigated are poly(alkyl isocyanate) with a butyl or hexyl side group. Polyisocyanates with an aromatic group on the nitrogen of main chain have been much less studied because the poly(aromatic isocyanates) have been considered not to take a rigid rodlike structure. Poly(benzyl isocyanate) is also not studied because it is insoluble in usual organic solvents. $^{1,2}$

It has been shown that achiral monomers such as triphenylmethyl methacrylate (TrMA), ${ }^{9-12}$ chloral, ${ }^{13,14}$ and $t$-butyl isocyanide ${ }^{15}$ yield optically active polymers with stable one- handed helical conformation when polymerized with optically active initiators. Therefore, these polymers are separable into right- and left-handed helices by liquid chromatography on chiral stationary phases. On the other hand, the helix reversal of poly(alkyl isocyanates) is a very fast process which does not allow the separation of rightand left-handed helices. ${ }^{5-8}$ This is one of the interesting properties of polyisocyanates.

In the present study, we carried out the polymerization of 3-phenylpropyl isocyanate, 4-phenylbutyl isocyanate, and butyl isocyanate by using optically active anionic initiators such as $t$ - BuLi-(-)-Sp and chiral lithium alkoxides or amides. The former will produce the polymer which contains no chiral fragment in the main chain but might be optically active if the polymer could have a stable one-handed helicity as observed in case of the polymer of TrMA. The latter initiators should give the polymer which contains a chiral 
fragment attaching to an initial chain end ( $\alpha$-end) and may be optically active if the polymer has a prevailing helicity induced by the chiral initiator fragment.

\section{EXPERIMENTAL}

3-Phenylpropyl isocyanate (PPI) was prepared by reaction of 3-phenylpropyl amine with phosgene under reflux in dry toluene, and purified by distillation under reduced pressure $\left(75-77^{\circ} \mathrm{C} / 0.35 \mathrm{mmHg}\right.$ ). Yield: $65 \%$. IR (neat, $\left.\mathrm{cm}^{-1}\right): 1605(\mathrm{C}=\mathrm{C}), 2282(\mathrm{~N}=\mathrm{C}=\mathrm{O}) \cdot{ }^{1} \mathrm{H}$ NMR $\delta\left(\mathrm{CDCl}_{3}, \mathrm{ppm}\right): 7.1-7.4(\mathrm{~m}, 5 \mathrm{H}), 3.3$ $(\mathrm{t}, 2 \mathrm{H}), 2.7(\mathrm{t}, 2 \mathrm{H}), 1.9(\mathrm{~m}, 2 \mathrm{H})$.

4-Phenylbutyl isocyanate (PBI) was prepared by the same method as described above $\left(90-95^{\circ} \mathrm{C} / 0.38 \mathrm{mmHg}\right)$. Yield: $78 \%$.

IR (neat, $\left.\mathrm{cm}^{-1}\right)$ : $1605 \quad(\mathrm{C}=\mathrm{C}), 2282$ $(\mathrm{N}=\mathrm{C}=\mathrm{O}) . \quad{ }^{1} \mathrm{H} \quad \mathrm{NMR} \quad \delta \quad\left(\mathrm{CDCl}_{3}, \mathrm{ppm}\right)$ : $7.1-7.4(\mathrm{~m}, 5 \mathrm{H}), 3.3(\mathrm{t}, 2 \mathrm{H}), 2.6(\mathrm{t}, 2 \mathrm{H})$, $1.5-1.8(\mathrm{~m}, 4 \mathrm{H})$.

Butyl isocyanate (BI) (Aldrich), PPI, and PBI were distilled over $\mathrm{CaH}_{2}$ under high vacuum just before polymerization.

Toluene and tetrahydrofuran (THF) were dried over $\mathrm{Na}$ wire and distilled onto $\mathrm{LiAlH}_{4}$. The solvents were distilled from $\mathrm{LiAlH}_{4}$ under high vacuum just before use.

\section{Preparation of Initiator Solution}

$(-)$-Sparteine (Sp) (Sigma), $(R)-(+)-N$-benzyl-1-phenylethyl amine $((+)$-BPEA) (Tokyo Kasei Kogyo (TCI)), (S)-(+)-1-(2-pyrrolidinylmethyl)pyrrolidine ((+)-PMP) (Aldrich), $(S)-(-)$-2-methoxymethylpyrrolidine $((-)$ MMP) (Merck), and (R)-(+)-3-dimethylaminopyrrolidine $((+)$-DAP) $(\mathrm{TCI})$ were purified by distillation over $\mathrm{CaH}_{2}$ under reduced pressure.

(-)-Menthol (TCI) and (-)-borneol (TCI) were used without further purification and (2S,3R)-(+)-4-dimethylamino-1,2-diphenyl3-methyl-2-butanol (Chirald) (Aldrich) was purified by recrystallization from ethanol.

The complex of tert-butyllithium with (-)-
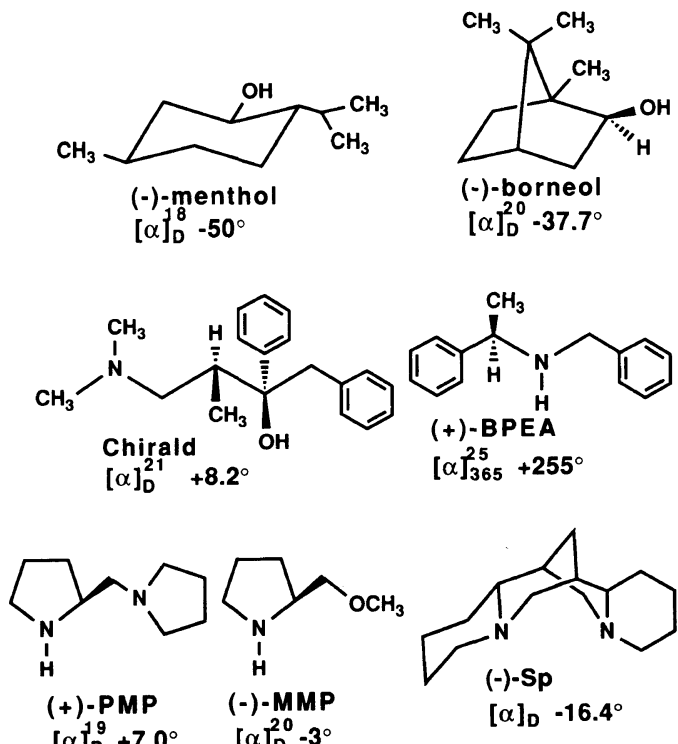

Figure 1. Optically active alcohols and amines used as initiators and ligand.

$\mathrm{Sp}(t-\mathrm{BuLi}-(-)-\mathrm{Sp})$ was prepared by mixing $(-)$-Sp with $t$-BuLi $([\mathrm{Sp}] /[t-\mathrm{BuLi}]=1.2)$ in toluene at room temperature. Lithium alkoxides of (-)-menthol, (-)-borneol, and Chirald and lithium amides of $(+)$-BPEA, (+)-PMP, (-)-MMP, and (+)-DAP were prepared by adding equimolar $t$-BuLi to the solution of optically active alcohols or amines in toluene at room temperature.

\section{Polymerization Procedure}

A monomer $(0.5 \mathrm{~g})$ and solvent $(5 \mathrm{ml})$ were placed in an ampule with a syringe and the solution was cooled to the prescribed temperature. Then, the above initiator solution was added to the monomer solution with a syringe. Gelation took place in a few seconds or minutes. The polymerization was terminated with a small amount of methanol and the mixture was kept for an hour at the temperature for complete termination. The product was precipitated in a large amount of methanol, filtered, and then dried in vacuo at $50^{\circ} \mathrm{C}$. 


\section{Measurements}

Gel permeation chromatography (GPC) was performed on a TOSOH HLC802A chromatograph equipped with a TSK-G3000H and a TSK-G5000H GPC columns. A calibration curve was obtained with poly(hexyl isocyanate) standards. ${ }^{16}$ Optical rotation was measured on a JASCO DIP-181 polarimeter. Circular dichroism (CD) spectra were measured on a JASCO J-720 spectrometer. IR spectra were recorded on a JASCO FT-IR-7000 infrared spectrophotometer. ${ }^{1} \mathrm{H}$ NMR spectra were measured on a Varian Gemini-200 $(200 \mathrm{MHz})$ spectrometer at $23^{\circ} \mathrm{C}$ and tetramethylsilane was used as internal standard.

\section{RESULTS AND DISCUSSION}

\section{Polymerization with $t-B u L i-(-)-S p$}

PPI was polymerized with $t$-BuLi-(-)-Sp in toluene at $-78^{\circ} \mathrm{C}$. Although a polymer was obtained in a high yield, it was insoluble in usual organic solvents such as $\mathrm{CHCl}_{3}, \mathrm{THF}$, DMF, etc. In order to obtain a soluble polymer and to evaluate a chiroptical feature of the polymer, PPI was copolymerized with BI by $t$-BuLi-(-)-Sp. The results of the copolymerization are collected in Table I. The copolymer obtained at a molar ratio of monomer in feed $[\mathrm{BI}] /[\mathrm{PPI}]=1 / 2$ was soluble in chloroform, while those obtained at lower $[\mathrm{BI}] /[\mathrm{PPI}]$ ratios were insoluble. The soluble copolymer showed

Table I. Copolymerization of PPI and BI with $t$-BuLi-(-)-Sp in toluene at $-78^{\circ} \mathrm{C}^{\mathrm{a}}$

\begin{tabular}{clc}
\hline BI/PPI & Yield $^{\mathrm{b}}$ & {$[\alpha]_{365}^{25}{ }^{\mathrm{e}}$} \\
\cline { 2 - 3 } & \multicolumn{1}{c}{} & \\
\hline $1 / 5$ & 43 & - \\
$1 / 3$ & 65 & - \\
$1 / 2$ & $82^{\mathrm{cd}}$ & 0 \\
\hline
\end{tabular}

a PPI, $0.5 \mathrm{~g} ;[\mathrm{M}] /[\mathrm{I}]=100$; toluene, $5 \mathrm{ml}$; time, $4 \mathrm{~h}$.

b Methanol-insoluble part.

c Soluble in $\mathrm{CHCl}_{3}$.

${ }^{d} \mathrm{BI} / \mathrm{PPI}$ in copolymer was $1 / 1.6$ by NMR.

e In $\mathrm{CHCl}_{3}$. no optical activity.

The results of the polymerization of PBI with $t$-BuLi- $(-)$-Sp are summarized in Table II. The obtained polymers were soluble in chloroform in contrast to the insolubility of poly(PPI) and poly(benzyl isocyanate). This indicates that the solubility of poly $(\omega$-phenylalkyl isocyanate) $\left(\mathrm{Ph}\left(\mathrm{CH}_{2}\right)_{n} \mathrm{NCO}\right)$ is dependent on the number of methylene $(n)$ and the polymer becomes soluble when the number is larger than 3 . The poly(PBI) was not optically active.

The poly(PBI) and poly(BI) must be helical polymers, but there exist left- and right-handed helical conformations with equal probability because of the absence of chiral components in the polymer molecules. Consequently, the polyisocyanates obtained with $t$-BuLi-(-)-Sp can not be optically active.

Table II. Polymerization of PBI with $t$-BuLi-(-)-Sp in toluene at $-78^{\circ} \mathrm{C}^{\mathrm{a}}$

\begin{tabular}{rccccc}
\hline$[\mathrm{M}] /[\mathrm{I}]$ & $\begin{array}{c}\text { Yield }^{\mathrm{b}} \\
\%\end{array}$ & $\begin{array}{c}\bar{M}_{w}^{\mathrm{c}} \\
\left(\times 10^{-3}\right)\end{array}$ & $\begin{array}{c}\bar{M}_{n}^{\mathrm{c}} \\
\left(\times 10^{-3}\right)\end{array}$ & $\bar{M}_{w} / \bar{M}_{n}^{\mathrm{c}}$ & {$[\alpha]_{365}^{25}{ }^{\mathrm{d}}$} \\
\hline 50 & 37 & 124 & 45 & 2.8 & 0 \\
100 & 47 & 113 & 28 & 4.1 & 0
\end{tabular}

a PBI. $0.5 \mathrm{~g}$; toluene, $5 \mathrm{ml}$; time, $4 \mathrm{~h}$.

b Methanol-insoluble part.

c By GPC.

d In $\mathrm{CHCl}_{3}$.

Table III. Polymerization of BI and PBI with $(+)$-BPEA lithium amide at $-78^{\circ} \mathrm{C}^{\mathrm{a}}$

\begin{tabular}{cccccr} 
No. & Monomer & Solvent & Yield $^{\mathrm{b}}$ & {$[\alpha]_{365}^{25}{ }^{\mathrm{c}}$} & $\begin{array}{c}\bar{M}_{n}{ }^{\mathrm{d}} \\
\left(\times 10^{-3}\right)\end{array}$ \\
\cline { 4 - 5 } & & & & \\
\hline 1 & BI & Toluene & 68 & -17 & 98 \\
2 & BI & THF & 40 & $-253^{\mathrm{e}}$ & 4 \\
3 & PBI & Toluene & 52 & -40 & 133 \\
4 & PBI & THF & 44 & -189 & 9
\end{tabular}

a Monomer, $0.5 \mathrm{~g}$; solvent, $5 \mathrm{ml}$; $[\mathrm{M}] /[\mathrm{I}]=50$; time, $4 \mathrm{~h}$.

b Methanol-insoluble part.

c In $\mathrm{CHCl}_{3}$.

d By GPC.

e $[\alpha]_{D}^{25} \sim-63^{\circ}$. 
Polymerization with Optically Active Lithium Alkoxides and Amides

Table III shows the results of the polymerization of BI and PBI with (+)-BPEA lithium amide in toluene or $\mathrm{THF}$ at $-78^{\circ} \mathrm{C}$. Optically active polymers were obtained from the two monomers in both the solvents. The polymers obtained in toluene had much higher molecular weight than those obtained in THF, although the yield of polymer was not quantitative in both the solvents. All the poly mers showed negative specific rotation which is opposite in sign to that of $(+)$-BPEA, indicating that the optical rotation of the polymers may be based on the prevailing helicity of the polymer chain caused by the chiral fragment of (+)-BPEA attaching to the polymer $\alpha$-end. The specific rotation of the polymers depended greatly on the molecular weight, and lower molecular weight polymers prepared in THF showed higher specific rotation. This indicates that the persistence length of helical structure induced by the chiral end group is not very long.

The oligomers isolated in the oligomerization of $m$-methylphenyl isocyanate with (-)-MMP lithium amide clearly indicated that each oligomer has an initiator fragment at the chain end. ${ }^{17}$

Figures 2 and 3 show UV and CD spectra of poly(BI) (No. 2 in Table III) and poly(PBI) (No. 4 in Table III) obtained in tetrahydrofuran (THF). The CD and UV spectral patterns of poly(BI) and poly(PBI) were similar to those of optically active helical poly(alkyl isocyanate)s. ${ }^{5}$ This indicates that optical activities of poly(BI) and poly(PBI) are due to the helical conformation of the main chain and $C D$ and UV absorptions of poly(PBI) result mainly from the amide units in the helical main chain. The intensities in CD and UV spectra of poly(PBI) were higher than those of poly(BI). The higher UV intensity for poly(PBI) seems to be due to the absorption of phenyl groups overlapping with that of amide units in main chain and higher CD intensity for poly(PBI)

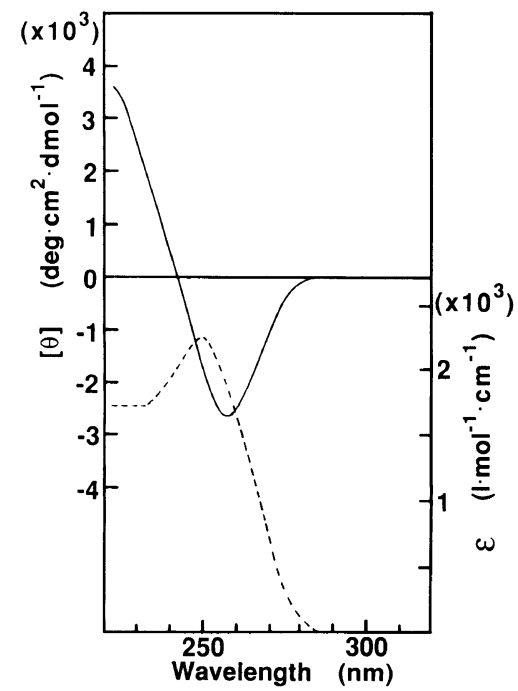

Figure 2. CD and UV spectra of poly(BI) (No. 2 in Table III) obtained in THF.

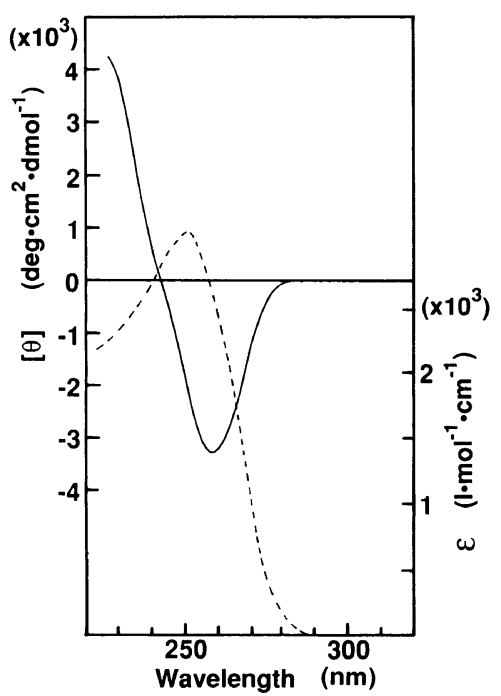

Figure 3. $\mathrm{CD}$ and UV spectra of poly(PBI) (No. 4 in Table III) obtained in THF.

may be ascribed to a longer persistence length of helix with one-screw sense of this polymer.

The results of the polymerization of BI with various optically active initiators are summarized in Table IV. Lithium alkoxides gave the polymers with higher molecular weight in higher yield than lithium amides. The specific rotations of the polymers obtained with the 
Table IV. Polymerization of BI in THF at $-78^{\circ} \mathrm{C}^{\mathrm{a}}$

\begin{tabular}{|c|c|c|c|c|c|c|}
\hline No. & Initiator & $\frac{\text { Yield }^{\mathrm{b}}}{\%}$ & {$[\alpha]_{365}^{25} \mathrm{c}$} & $\begin{array}{c}\bar{M}_{w}{ }^{\mathrm{d}} \\
\left(\times 10^{-3}\right)\end{array}$ & $\begin{array}{c}\bar{M}_{n}^{\mathrm{d}} \\
\left(\times 10^{-3}\right)\end{array}$ & $\bar{M}_{w} / \bar{M}_{n}^{\mathrm{d}}$ \\
\hline 1 & Li-menthoxide & 79 & +4.7 & 107 & 51 & 2.1 \\
\hline 2 & Li-borneoxide & 53 & -12 & 59 & 25 & 2.4 \\
\hline $3^{\mathrm{e}}$ & Li-borneoxide & 9 & -35 & 6 & 3 & 1.8 \\
\hline 4 & Li-Chirald & 77 & -31 & 114 & 21 & 5.3 \\
\hline 5 & Li-PMP & 20 & +415 & 35 & 9 & 4.0 \\
\hline 6 & Li-MMP & 10 & +416 & 31 & 9 & 3.3 \\
\hline
\end{tabular}

a BI, $0.5 \mathrm{~g}$; THF, $5 \mathrm{ml} ;[\mathrm{M}] /[\mathrm{I}]=50$; time, $4 \mathrm{~h}$.

b Methanol-insoluble part.

c In $\mathrm{CHCl}_{3}$.

d By GPC.

e $[\mathrm{M}] /[\mathrm{I}]=8$.
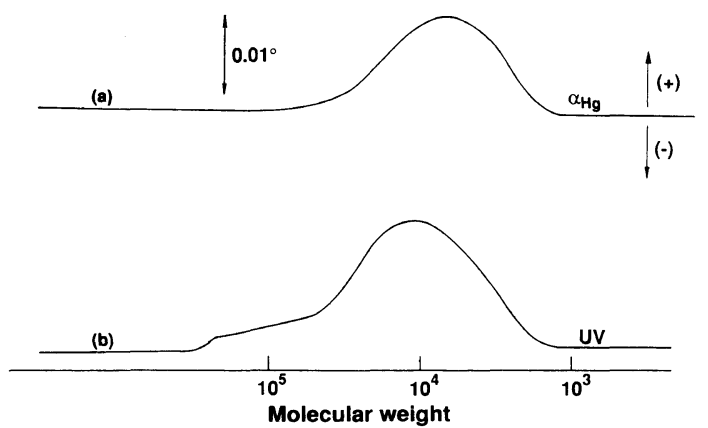

Figure 4. GPC curve of poly(BI) (No. 6 in Table IV) measured with polarimeter (a) and UV $(270 \mathrm{~nm})$ detectors (b).

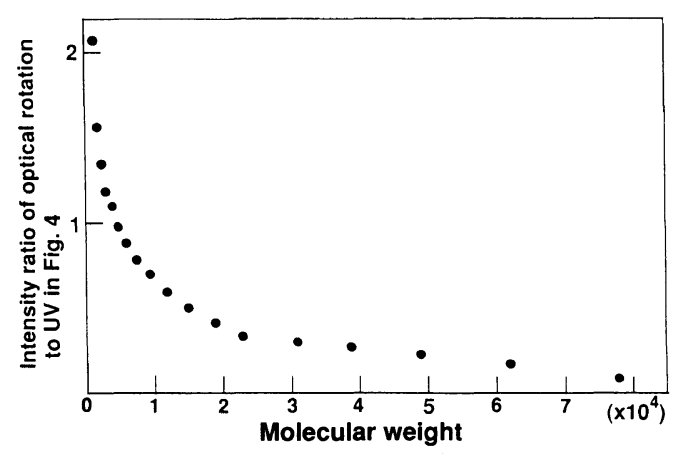

Figure 5. Plots of optical rotation vs. molecular weight of poly(BI) shown in Figure 4.

optically active amide initiators were much greater than those with the optically active alkoxides. Probably sterically crowded second-

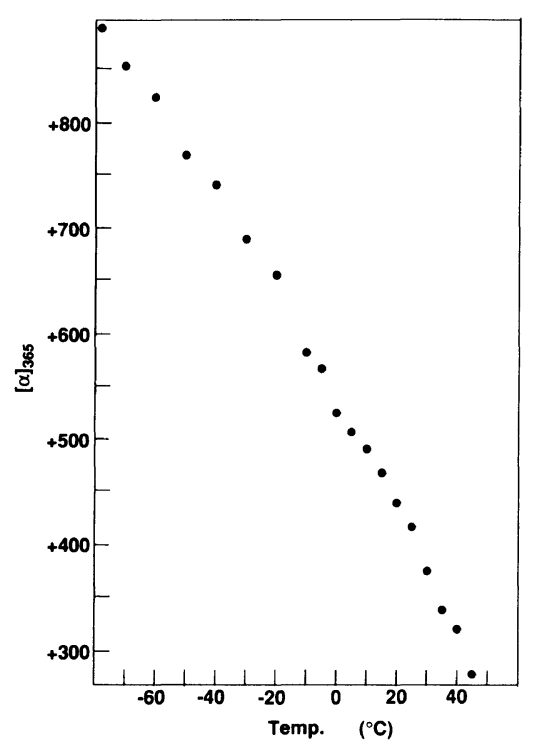

Figure 6. Dependence of specific rotation of poly(BI) (No. 5 in Table IV) on temperature (solvent, chloroform-toluene $(2: 1))$.

ary amino groups can show more efficient twisting power for controlling screw sense of the polymer than the flexible alkoxy groups, even if we take into account the difference in molecular weight of the polymers. The unimer obtained with (-)-MMP lithium amide showed negative specific rotation at $589 \mathrm{~nm}$ which is opposite in sign to that of the polymer and the same as that of (-)-MMP, indicating 
that the optical rotation of the polymer may be based on the prevailing helicity of the polymer chain.

To elucidate the molecular weight dependence of optical rotation of poly(BI) (No. 6 in Table IV), GPC measurement was performed with UV and polarimetric detectors (Figure 4). The relative intensity ratio of optical rotation to UV absorbance, which approximately corresponds to the specific rotation of the polymer, was plotted against molecular weight (Figure 5). The optical rotation seems to dramatically decrease with an increase in molecular weight of the polymer.

The optical rotation of poly(BI) (No. 5 in Table IV) also depended greatly on the temperature. Figure 6 shows the change of specific rotation at various temperatures measured in a mixture of chloroform and toluene $(2: 1)$ under nitrogen atmosphere. The specific rotation increased with a decrease in temperature. Similar temperature dependence of optical rotation has been reported for optically active polyisocyanates. ${ }^{5-8}$ The temperature dependence of optical activity may be because at lower temperature, the chiral initiator fragment attaching to the polymer $\alpha$-end can exert its twisting power more efficiently to lead the polymer conformation with greater one-handed helicity and also persistence length of the polymer chain may increase.

Acknowledgments. We are grateful to Professor Akio Teramoto and Dr. Takahiro Sato of Osaka University for providing the poly- (hexyl isocyanate) standards for the GPC calibration. A part of this study was supported by the Asahi Glass Foundation and a Grant-in-Aid for Scientific Research (No. 04403021) from the Ministry of Education, Science, and Culture of Japan.

\section{REFERENCES}

1. A. J. Bur and L. J. Fetters, Chem. Rev., 76, 727 (1976).

2. V. E. Shashoua, W. Sweeny, and R. F. Tietz, J. Am. Chem. Soc., 82, 866 (1960).

3. M. Goodman and S. Chen, Macromolecules, 3, 398 (1970).

4. M. Goodman and S. Chen, Macromolecules, 4, 625 (1971).

5. M. M. Green, C. Andreola, B. Munoz, and M. P. Reidy, J. Am. Chem. Soc., 110, 4063 (1988).

6. M. M. Green and M. P. Reidy, J. Am. Chem. Soc., 111, 6452 (1989).

7. S. Lifson, C. Andreola, N. C. Peterson, and M. M. Green, J. Am. Chem. Soc., 111, 8850 (1989).

8. M. M. Green, S. Lifson, and A. Teramoto, Chirality, 3, 285 (1991).

9. Y. Okamoto, K. Ohta, K. Hatada, and H. Yuki, $J$. Am. Chem. Soc., 101, 4763 (1979).

10. Y. Okamoto, K. Suzuki, and H. Yuki, J. Polym. Sci., Polym. Chem. Ed., 18, 3043 (1980).

11. Y. Okamoto, H. Shohi, and H. Yuki, J. Polym. Sci., Polym. Lett. Ed., 21, 601 (1983).

12. T. Nakano, Y. Okamoto, and K. Hatada, J. Am. Chem. Soc., 114, 1318 (1992).

13. L. S. Corley and O. Vogl, Polym. Bull., 3, 211 (1980).

14. K. Ute, K. Oka, Y. Okamoto, K. Hatada, F. Xi, and O. Vogl, Polym. J., 23, 1419 (1991).

15. P. C. J. Kamer, R. J. M. Nolte, and W. Drenth, $J$. Am. Chem. Soc., 110, 6818 (1988).

16. T. Itou, H. Chikiri, A. Teramoto, and S. M. Aharoni, Polym. J., 20, 143 (1988).

17. M. Matsuda, T. Nakano, and Y. Okamoto, Polym. Prepr. Jpn., 41, 2068 (1992). 